\title{
Intensity-modulated radiotherapy for synchronous cancer of the anal canal and cervix
}

\author{
TERUFUMI KAWAMOTO $^{1,2}$, KEI ITO ${ }^{1,2}$, TAKUYA SHIMIZUGUCHI ${ }^{1}$, \\ SATOSHI KITO $^{1}$, KEIJI NIHEI ${ }^{1}$, KEISUKE SASAI ${ }^{2}$ and KATSUYUKI KARASAWA ${ }^{1}$ \\ ${ }^{1}$ Division of Radiation Oncology, Tokyo Metropolitan Cancer and Infectious Diseases Center, \\ Komagome Hospital, Tokyo 113-8677; ${ }^{2}$ Department of Radiation Oncology, \\ Graduate School of Medicine, Juntendo University, Tokyo 113-8421, Japan
}

Received October 18, 2017; Accepted July 10, 2018

DOI: $10.3892 / \mathrm{ol} .2018 .9229$

\begin{abstract}
Due to recent advancements in diagnostic techniques, the incidence of multiple primary cancer has increased; however, synchronous cancer of the anal canal and cervix (SCACC) is rare, and no previous studies have investigated the treatment of this disease. The present study reports a case in which intensity-modulated radiotherapy (IMRT) was used to treat a 64-year-old female with SCACC, inguinal lymphadenopathy and anal pain. The patient was diagnosed with cT3N3M0 stage IIIb anal canal squamous cell carcinoma and cT1b1N0M0 stage Ib1 cervical squamous cell carcinoma, based on biopsy and imaging study data. According to the definitive treatment for advanced-stage anal canal cancer, outpatient treatment with chemoradiotherapy (CRT) using S-1 for SCACC was recommended, as the patient did not want to undergo resection of the anus. Considering the lymph node regions involved in SCACC and the necessary doses, the treatment plan was as follows: Whole pelvis and inguinal lymph node region radiation (36 Gy/20 fractions); a first booster radiation dose ( $9 \mathrm{~Gy} / 5$ fractions) for the whole pelvis; and a second booster radiation dose ( $14.4 \mathrm{~Gy} / 8$ fractions) for the primary lesions. The patient was prescribed $\mathrm{S}-1$ at a dose of $60 \mathrm{mg} / \mathrm{m}^{2} /$ day twice daily on days $1-14$ and 29-42. The patient experienced grade 2 diarrhea and anal mucositis, but CRT
\end{abstract}

Correspondence to: Dr Terufumi Kawamoto, Division of Radiation Oncology, Tokyo Metropolitan Cancer and Infectious Diseases Center, Komagome Hospital, 18-22-3 Honkomagome, Tokyo 113-8677, Japan

E-mail: t.kawamoto@cick.jp

Abbreviations: MPC, multiple primary cancer; SCACC, synchronous cancer of the anal canal and cervix; CRT, chemoradiotherapy; RT, radiotherapy; IMRT, intensity-modulated radiotherapy; HDR-ICBT, high-dose-rate intracavitary brachytherapy; EBRT, external beam radiotherapy; RCT, randomized clinical trial

Key words: synchronous primary cancer, anal canal cancer, cervical cancer, squamous cell carcinoma, S-1 was completed without discontinuation and hospitalization. The patient exhibited a complete response and remained disease-free without any treatment-associated complications at the 6-month follow-up. In conclusion, SCACC was successfully treated with IMRT in the present case. It is important to determine the treatment strategy for synchronous cancer types, taking into consideration the tumor stage, tumor location and patient situation.

\section{Introduction}

Due to recent advancements in diagnostic techniques, the incidence of patients with multiple primary cancer (MPC) has increased (1). Evidence suggests that the prevalence of MPC is between 0.73 and $17 \%$ globally from 1966 to 2015 (2-7). Different values were reported in the literature on the prevalence of MPC, this is due to the long evaluation period and the inclusion of autopsy series; therefore, there were differences between the studies (2-4,7) The risk of developing MPC varies between different cancer sites and is reported in a range from $1 \%$ (primary liver malignancy) up to $16 \%$ (primary bladder cancer) (4). MPC reflects not only the late effects of therapy but also the influence of shared etiologic factors (in particular, tobacco and excessive alcohol intake), genetic susceptibility, environmental exposures, host effects, and combinations of factors, including gene-environment interactions (8). Risks for selected MPC are also modified by age at exposure and attained age (9). For simplicity, Travis et al (10) categorized MPC into three major groups according to the predominant etiologic factor: Treatment-related, syndromic, and those due to shared etiologic influences; however, they underscored the nonexclusivity of these groups.

Human papilloma viruses 16 and 18 have been implicated in the development of synchronous cancer of the anal canal and cervix (SCACC), although this disease is rare. According to National Cancer Center data, there were 123 patients with MPC (2.7\%) among 4,480 patients with cervical cancer between 1962 and 1996, but there were no reports of patients with anal canal cancer (11). The standard treatment for locally advanced anal canal cancer is chemoradiotherapy (CRT), and for early-stage cervical cancer the standard treatment is surgery or radiotherapy (RT); however, to the best of our 
knowledge, no previous studies have reported on the treatment of SCACC. The present study reports a case of SCACC treated with intensity-modulated RT (IMRT).

\section{Case report}

A 64-year-old female presented with inguinal lymphadenopathy and anal pain. The patient was admitted to Komagome Hospital (Tokyo, Japan) in January 2017. The patient had undergone total mastectomy for left breast cancer at the age of 42 years and cervical conization for cervical cancer (cervical intraepithelial neoplasia) at the age of 46 years, but they had no family history of cancer. The patient smoked one pack of cigarettes per day and drank socially, and they also had a history of frequent sexual intercourse with multiple sexual partners, but no anal intercourse. Laboratory tests determined elevated serum squamous cell carcinoma (SCC) antigen, cytokeratin 19 fragment and cancer antigen 19-9 levels, and the patient tested negative for human immunodeficiency virus. Magnetic resonance imaging of the pelvis depicted a malignant stricture in the anal canal that extended $55 \mathrm{~mm}$ superiorly from the anal verge, and a malignant stricture in the cervix, with a diameter of up to $25 \mathrm{~mm}$ (Fig. 1). Computed tomography (CT) and positron emission tomography indicated no evidence of distant metastases, and the pararectal, left inguinal and bilateral iliac lymph nodes were suspected to exhibit tumor involvement. Pelvic examination demonstrated a 25-mm ulceroproliferative growth in the cervix. Rectal examination indicated an ulceroproliferative growth at the anal verge, extending up to $20 \mathrm{~mm}$. In the two lesions, micro-photographs showed sheets and nests of tumor cells separated by fibrous septae. The tumor cells showed squamoid differentiation. Therefore, biopsy diagnosed SCC, and increased p16 expression was determined by immunohistochemistry (Fig. 2). Immunohistochemical staining for p16 was performed as follows (Bond Max Protocol; $3 \mathrm{~mm}$-thick sections were prepared from $10 \%$ formalin fixed at ambient temperature for $24 \mathrm{~h}$, paraffin-embedded tissue blocks. Tissue sections were deparaffinized with Bond Dewax Solution (Leica Microsystems, Ltd., Milton Keynes, UK) according to the manufacturer's protocol at $72^{\circ} \mathrm{C}$ for $30 \mathrm{~min}$. Tissue sections were pretreated with the epitope retrieval (Bond Epitope Retrieval Solution 2 contained an EDTA based buffer and surfactant, $\mathrm{pH} 8.8$ ) at $100^{\circ} \mathrm{C}$ for $10 \mathrm{~min}$. Following washing, peroxidase blocking with $3 \%$ Hydrogen peroxide was performed at ambient temperature for $10 \mathrm{~min}$. The sections were again washed with Bond Wash Solution (Leica Microsystems, Ltd.) at ambient temperature for $6 \mathrm{~min}$ and then incubated with the p16 primary antibody (Ventana Medical Systems; cat no. 705-4713; Tucson, AZ) at ambient temperature for $15 \mathrm{~min}$. The p16 primary antibody was diluted in $0.05 \mathrm{M}$ Tris- $\mathrm{HCl}$ with $1 \%$ carrier protein, and $0.10 \%$ ProClin 300 , a preservative. The sections were incubated with Post Primary reagent [Rabbit anti mouse $\mathrm{IgG}$ with $10 \%$ (v/v) animal serum in tris-buffered saline/0.09\% ProClin ${ }^{\mathrm{TM}}$ 950; cat no. DS9800; Leica Microsystems, Ltd.] for $8 \mathrm{~min}$ at ambient temperature, followed by washing using Bond Wash solution (Leica Microsystems, Ltd.) for $6 \mathrm{~min}$, and BondTM Polymer Refine Detection (anti-rabbit Poly-HRP-IgG; cat no. DS9800; Leica Microsystems, Ltd.) according to the manufacturer's protocol and placed on the slides for $8 \mathrm{~min}$ at ambient temperature, followed by washing using Bond Wash solution (Leica Microsystems, Ltd.) and distilled water for $4 \mathrm{~min}$. The sections were developed with 3,39-diaminobenzidine-chromogen at ambient temperature for $10 \mathrm{~min}$, and counterstained with hematoxylin at ambient temperature for $5 \mathrm{~min}$. Stained tissues were viewed and images captured using a light microscope under five fields in each sample (magnification, x100). Therefore, cT3N3M0 stage IIIb anal canal cancer (UICC 2010) and cT1b1N0M0 stage Ib1 (UICC 2010) cervical cancer were diagnosed (12).

According to the definitive treatment for advanced-stage anal canal cancer, outpatient treatment with CRT (59.4 Gy/33 fractions) using S-1 without using high-dose-rate intracavitary brachytherapy (HDR-ICBT) for SCACC was recommended, as the patient did not want to undergo resection of the anus.

In order to reduce the dose to the organs at risk (OARs), such as the small bowel, RT was delivered via a three-step IMRT technique. Considering the lymph node region affected by SCACC and the necessary doses, step 1 included the delivery of $36 \mathrm{~Gy}$ in 20 fractions to the whole pelvis, the inguinal lymph node region and the involved primary lesions. Step 2 included the delivery of an additional 9 Gy in 5 fractions to the whole pelvis and the involved primary lesions. Finally, step 3 included the delivery of an additional 14.4 Gy in 8 fractions to the primary lesions (Table I). The prescribed dose was defined as $50 \%$ of the planning target volume that should receive $100 \%$ of the dose. OARs were contoured, including the peritoneal space (bowel bag), small bowel loops, bladder and bilateral femoral head. Table II summarizes the dose constraints for the OARs. Depending on the extent of the tumor, it was permissible to exceed the dose constraints to the bowel bag and small bowel loops. Fig. 3 describes the dose-distribution and dose-volume histogram. The patient was prescribed S-1 at a dose of $60 \mathrm{mg} / \mathrm{m}^{2} /$ day twice daily on days 1-14 and days 29-42, and treatment was tolerated the well; however, Common Terminology Criteria for Adverse Events (v4.0), including grade 2 diarrhea and anal mucositis (13), were demonstrated. CRT was completed without discontinuation through outpatient treatment.

Following treatment, no tumors were detected by imaging studies or pathological examinations (Fig. 4). At the 15 months total follow-up, the patient continued to be disease-free, without any treatment-associated complications.

\section{Discussion}

The present report describes a rare case of SCACC that was successfully treated with IMRT. The current criteria for the diagnosis of MPC, which were defined by Warren and Gates (14), are as follows: i) Each lesion must be malignant; ii) each lesion must exhibit a distinctively different pathology; and iii) metastases from prior malignancies must be excluded. Among patients with MPC, double cancer is primarily observed, with triple cancer occurring in $0.5 \%$ of patients, and quadruple or quintuple cancer occurring in $<0.1 \%$ of patients (15). MPC can be divided into two categories depending on the interval between each diagnosis: Synchronous cancer is a secondary cancer occurring simultaneously or within 6 months of the first malignancy; 
Table I. Target volumes.

\begin{tabular}{|c|c|c|}
\hline Steps & Dose, Gy & Targets \\
\hline Step 1 & 36 & $\begin{array}{l}\text { Primary lesions (anal canal tumor, cervical tumor, metastatic pararectal node, left inguinal node and } \\
\text { bilateral iliac nodes) } \\
\text { Whole pelvis (mesorectum, parametrium, presacral space, common external and internal iliac lymph } \\
\text { node regions, and obturator lymph node region) } \\
\text { Inguinal lymph node region }\end{array}$ \\
\hline Step 2 & 9 & Primary lesions and whole pelvis \\
\hline Step 3 & 14.4 & Primary lesions \\
\hline
\end{tabular}
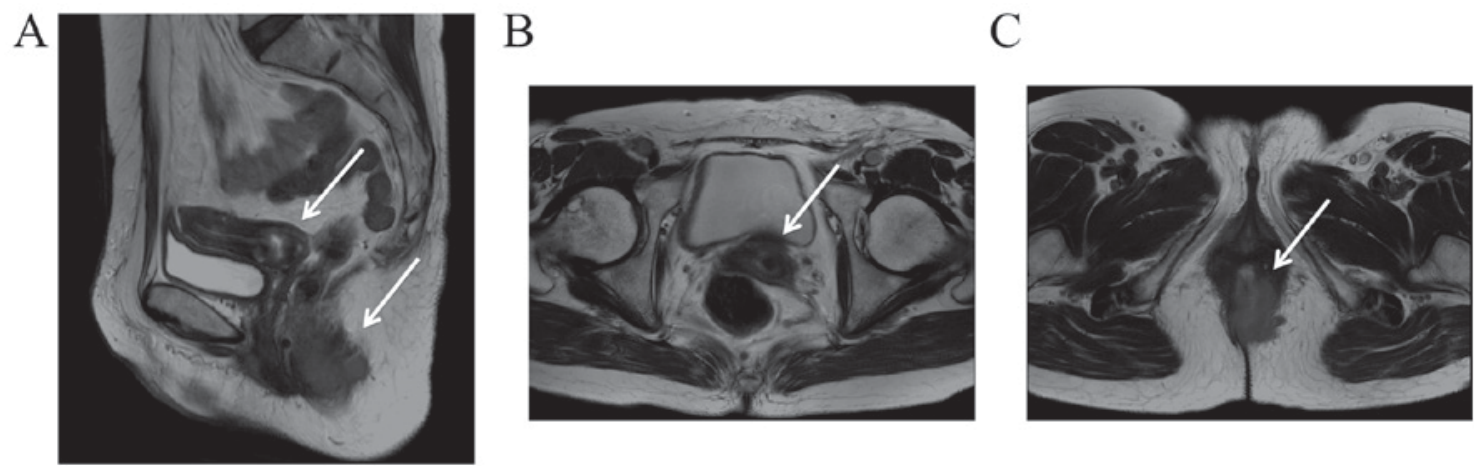

Figure 1. (A) Sagittal and (B and C) axial slices of T2-weighted magnetic resonance images. The tumor was located in the anal canal and cervix (arrows).
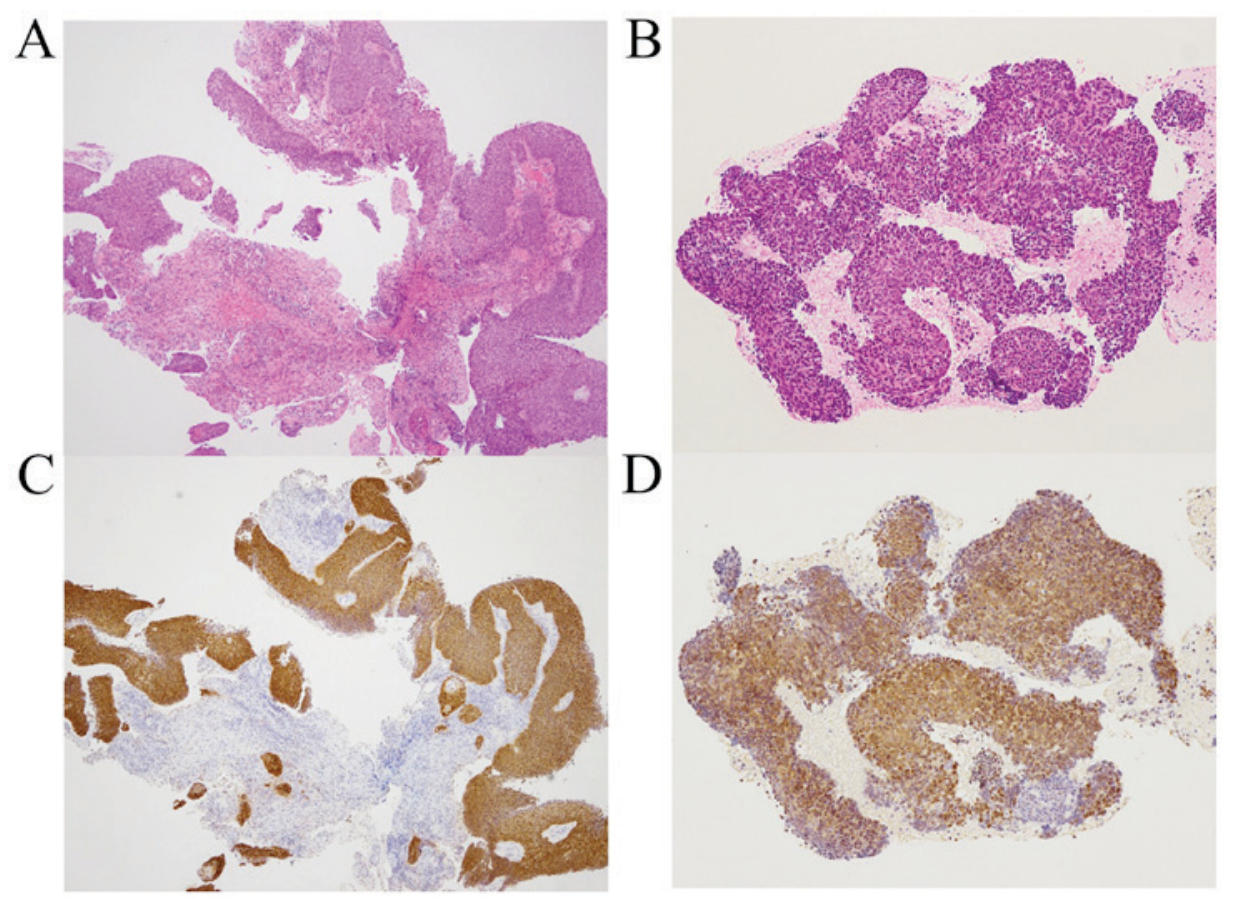

Figure 2. Histological examination of the anal canal and cervix. Histological examination demonstrated that the cancer was squamous cell carcinoma in the (A) anal canal and (B) cervix following hematoxylin and eosin staining. Increased p16 expression in the (C) anal canal and (D) cervix was detected by immunohistochemistry.

whereas, metachronous cancer is a secondary cancer that develops $>6$ months after the first malignancy (16). In the present case, the anal canal and cervical tumors contained intraepithelial components and the two tumors were discontinuous. The patient had a medical history of left-sided breast cancer. Based on these data, the patient was diagnosed with double primary synchronous and triple primary metachronous cancer.

Generally, the treatments for synchronous cancer remain unclear. It is vital to determine the treatment strategy for 
Table II. Dose constraints for organs at risk.

\begin{tabular}{ll}
\hline Organs & \multicolumn{1}{c}{ Desired value } \\
\hline Bowel bag & $\mathrm{V}_{15 \mathrm{~Gy}}<830 \mathrm{ml} \mathrm{V} 25 \mathrm{~Gy}$ \\
and $\mathrm{V}_{45 \mathrm{~Gy}}<650 \mathrm{ml}$, \\
Small bowel loops & $\mathrm{V}_{15 \mathrm{~Gy}}<275 \mathrm{ml}, \mathrm{V}_{25 \mathrm{~Gy}}<190 \mathrm{ml}$, \\
& and $\mathrm{V}_{45 \mathrm{~Gy}}<120 \mathrm{ml}$ \\
Bladder & $\mathrm{V}_{45 \mathrm{~Gy}}<70 \%$ organ volume \\
Femoral head & $\mathrm{V}_{50 \mathrm{~Gy}}<10 \%$ organ volume
\end{tabular}

V, volume.

synchronous cancer, considering the tumor stage, tumor location and patient situation (17). Based on the definitive treatment for advanced-stage anal canal cancer, the present treatment policy involved concurrent therapy for SCACC, as the tumors were in close proximity. Table III depicts the comparisons of treatments for anal canal and cervical cancer.

In previous studies, in patients with locally advanced anal canal cancer, the combination of RT and infused 5-fluorouracil (5-FU) and mitomycin resulted in a significantly improved locoregional control rate and a reduction in the requirement for colostomy, without a significant increase in late side effects, compared with RT alone (18). In patients with T3-, T4- or lymph node-positive anal canal cancer, $\mathrm{RT}$ doses of $\geq 54$ Gy administered with limited treatment breaks ( $<60$ days) were associated with increased locoregional control (19). The effect of further escalation of radiation doses was assessed in the ACCORD 03 trial, which indicated that doses of $>60$ Gy provided no additional benefit to patients with anal canal cancer (20). In patients with early-stage cervical cancer, a randomized clinical trial (RCT) demonstrated no significant difference in the overall survival between patients treated with surgery and those treated with definitive RT (21); therefore, definitive RT without chemotherapy has been accepted as a treatment option for early-stage cervical cancer. In Japan, definitive RT comprising of whole pelvis external beam RT (EBRT) of $20 \mathrm{~Gy} / 10 \mathrm{frac}-$ tions, pelvic EBRT with a midline block of $30 \mathrm{~Gy} / 15$ fractions and HDR-ICBT of $24 \mathrm{~Gy} / 4$ fractions at point A [biologically effective dose (BED), $62.4 \mathrm{~Gy}_{10}$ ] was a safe and effective treatment for patients with stage I and II cervical cancer with a small $(<4 \mathrm{~cm})$ tumor diameter (22). Based on these reports, it was considered that EBRT doses of $59.4 \mathrm{~Gy} / 33$ fractions for primary lesions (BED, $70.1 \mathrm{~Gy}_{10}$ ) could be applied for the two primary tumors without HDR-ICBT. Therefore, in the present study EBRT doses of 59.4 Gy/33 fractions without HDR-ICBT were used.

The lymph node region involved in anal canal cancer is similar to that in cervical cancer, but anal canal cancer involves the inguinal lymph node region and cervical cancer involves the common iliac lymph node region (23). Considering comprehensive regional nodal irradiation to each area, IMRT was adopted in the present study in order to reduce the dose to the OARs. With regard to the effectiveness of IMRT, the results of a phase II trial for anal canal cancer treated with IMRT demonstrated that the incidence of acute

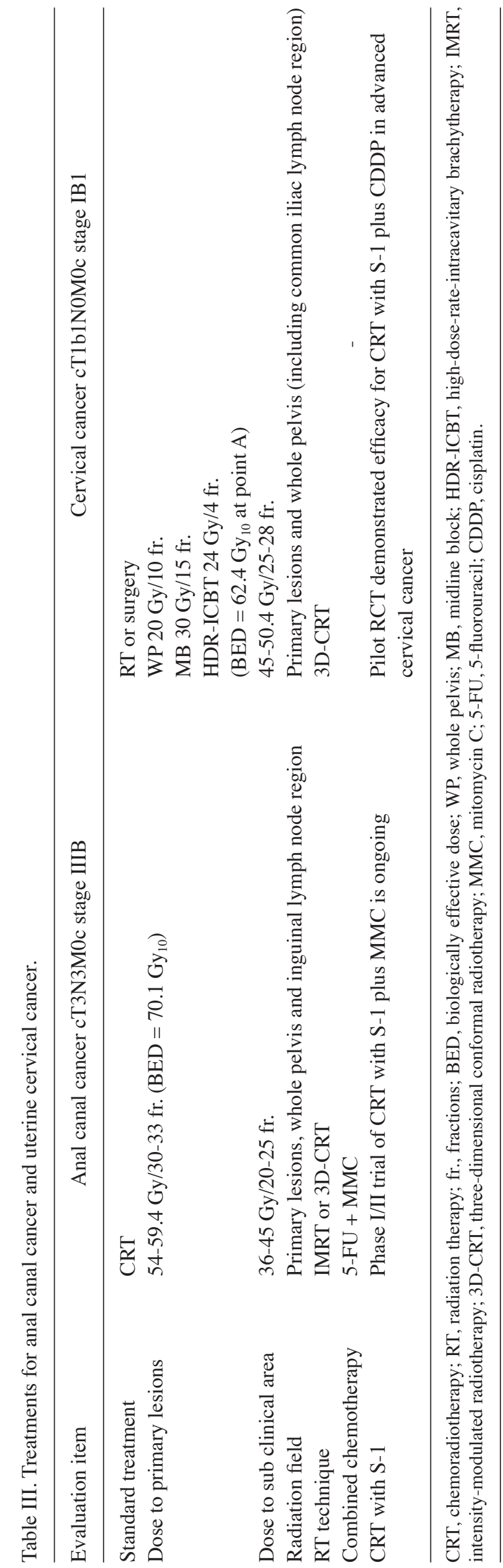


A

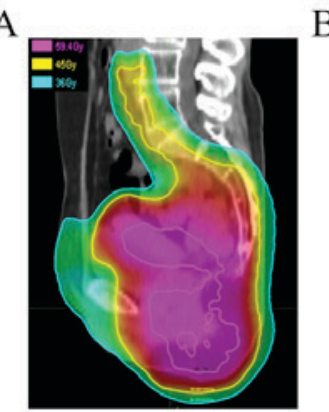

B

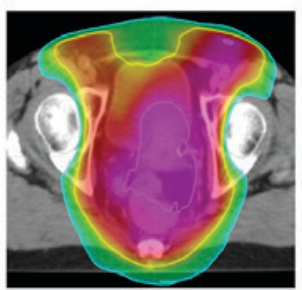

C

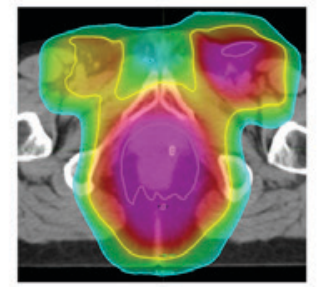

D

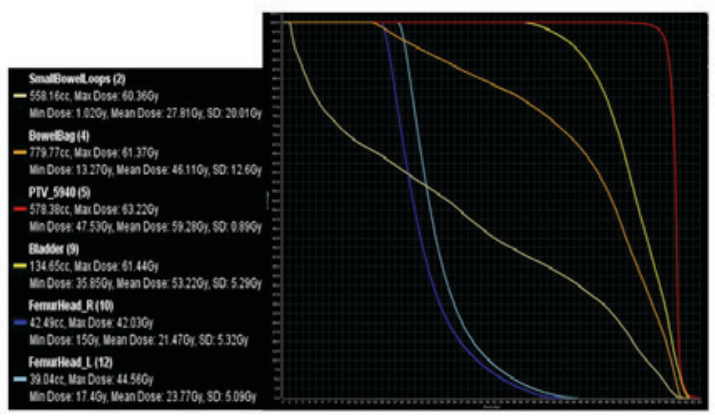

Figure 3. Dose distribution and dose-volume histogram. (A) Sagittal and (B and C) axial slices depicting representative intensity-modulated radiotherapy dose distributions for squamous cell carcinoma. (D) Dose-volume histograms depicting the dosimetric parameters analyzed at the PTV, bladder, bowel bag, small bowel loops, left femoral head and right femoral head. SD, standard deviation; PTV, planning target volume.

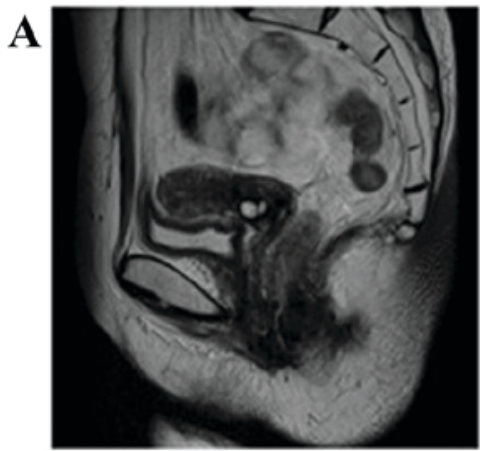

B

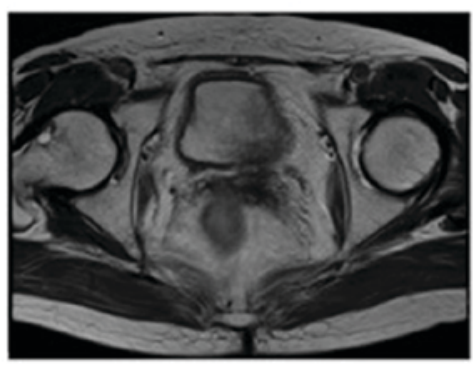

C

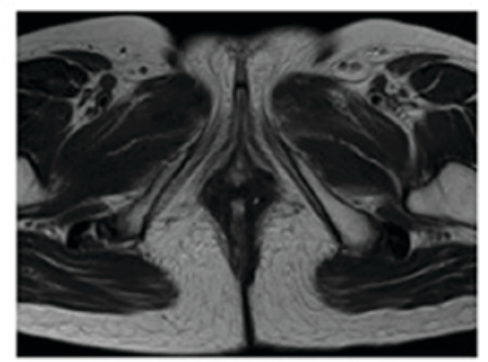

Figure 4. (A) Sagittal and (B and C) axial slices of T2-weighted magnetic resonance images. Magnetic resonance imaging following chemoradiotherapy demonstrated a complete response.

gastrointestinal morbidity of at least grade 3 was significantly lower, compared with previous three-dimensional conformal RT(21 vs. $37 \% ; \mathrm{P}=0.005)(24,25)$. Conversely, the effectiveness of IMRT for cervical cancer remains controversial due to uterine motion (26). To account for internal uterine motion, the following steps were taken in the present study: Firstly, to ensure reproducibility, the patient was instructed to empty their bladder and consume $500 \mathrm{cc}$ of water $30 \mathrm{~min}$ prior to the scan and treatment; secondly, to ensure reproducibility, magnesium oxide for controlling intestinal function and a laxative to maintain regular bowel function and an empty rectum was administered; thirdly, the internal target volume was defined as the uterine clinical target volume with $10-\mathrm{mm}$ anteroposterior, 10-mm superoinferior and 5-mm lateral margins, according to a previous report (27) and finally, the treatment position was verified using daily megavoltage CT imaging. As a result, the patient achieved a complete response without diarrhea or anal mucositis of grade 3 or higher.

With regard to chemotherapy, $\mathrm{S}-1$ is an orally administered antitumor drug composed of tegafur, 5-chloro-2,4-dihydroxypyridine and oteracil potassium. S-1 results in an increase in radiosensitivity of the tumor cells, as reported in a previous study (28). Clinical trials of $\mathrm{S}-1$ have been performed for various cancer types. S-1 is not inferior to 5-FU in a superiority style, and in view of the convenience of oral administration, $\mathrm{S}-1$ could replace intravenous 5-FU for the treatment of unresectable or recurrent gastric cancer (29). According to these data, a phase I/II trial of CRT with S-1 plus mitomycin $\mathrm{C}$ is ongoing in patients with stage II/III squamous cell carcinoma of the anal canal (30). In advanced cervical cancer, the results of a pilot RCT demonstrated promising efficacy and an acceptable toxicity of CRT with S-1 plus cisplatin, compared with CRT with cisplatin alone (31). Based on these reports, it was considered that S-1 may be an effective treatment for SCACC and recommended for outpatient treatment.

Increased p16 expression has been demonstrated to be an important prognostic determinant for patients with anal canal and cervical cancer treated with RT $(32,33)$. In the present case, although the definitive treatment was lacking in terms of combination chemotherapy without 5-FU and mitomycin for anal canal cancer and an irradiation method without ICBT for cervical cancer, the promising treatment results may have been reflected by the increased p16 expression simultaneously observed in each tumor.

In conclusion, a rare case of SCACC that was successfully treated with IMRT is reported in the present study. It is important to determine the treatment strategy for synchronous cancer, taking into consideration the tumor stage, tumor location and patient situation.

\section{Acknowledgements}

Not applicable.

\section{Funding}

No funding was received. 


\section{Availability of data and materials}

All data generated or analyzed during this study are included in this published article.

\section{Authors' contributions}

TK prepared the manuscript and the literature search. TK, KI and TS contributed to analysis and interpretation of data, and assisted in the preparation of the manuscript. SK, KN, KS and KK have contributed to data collection and interpretation, and critically reviewed the manuscript. All authors approved the final version of the manuscript, and agree to be accountable for all aspects of the work in ensuring that questions related to the accuracy or integrity of any part of the work are appropriately investigated and resolved.

\section{Ethics approval and consent to participate}

Not applicable.

\section{Patient consent for publication}

The patient provided written informed consent for publication of this case report, and the privacy policy was fully explained.

\section{Competing interests}

The authors declare that they have no competing interests.

\section{References}

1. Kanguru L, Bikker A, Cavers D, Barnett K, Brewster DH, Weller D and Campbell C: Pathways to diagnosis of a second primary cancer: Protocol for a mixed-methods systematic review. BMJ Open 7: e017929, 2017.

2. Spratt JS Jr and Hoag MG: Incidence of multiple primary cancers per man-year of follow up: 20-year review from the Ellis Fischel State Cancer Hospital. Ann Surg 164: 775-784, 1966.

3. Demandante CG, Troyer DA and Miles TP: Multiple primary malignant neoplasms: Case report and a comprehensive review of the literature. Am J Clin Oncol 26: 79-83, 2003.

4. Hayat MJ, Howlader N, Reichman ME and Edwards BK: Cancer statistics, trends, and multiple primary cancer analyses from the surveillance, epidemiology, and end results (SEER) program. Oncologist 12: 20-37, 2007.

5. Coyte A, Morrison DS and McLoone P: Second primary cancer risk-the impact of applying different definitions of multiple primaries: Results from a retrospective population-based cancer registry study. BMC cancer 14: 272, 2014.

6. Utada M, Ohno Y, Hori M and Soda M: Incidence of multiple primary cancers and interval between first and second primary cancers. Cancer Sci 105: 890-896, 2014.

7. Molina-Montes E, Requena M, Sánchez-Cantalejo E, Fernández MF, Arroyo-Morales M, Espín J, Arrebola JP and Sánchez MJ: Risk of second cancers cancer after a first primary breast cancer: A systematic review and meta-analysis. Gynecol Oncol 136: 158-171, 2015.

8. Travis LB: Therapy-associated solid tumors. Acta Oncol 41: 323-333, 2002.

9. Travis LB, Fosså SD, Schonfeld SJ, McMaster ML, Lynch CF, Storm H, Hall P, Holowaty E, Andersen A, Pukkala E, et al: Second cancers among 40,576 testicular cancer patients: Focus on long-term survivors. J Natl Cancer Inst 97: 1354-1365, 2005.

10. Travis LB, Rabkin CS, Brown LM, Allan JM, Alter BP, Ambrosone $\mathrm{CB}$, Begg $\mathrm{CB}$, Caporaso $\mathrm{N}$, Chanock S, DeMichele A, et al: Cancer survivorship-genetic susceptibility and second primary cancers: Research strategies and recommendations. J Natl Cancer Inst 98: 15-25, 2006.
11. Kaneko S and Yamaguchi N: Epidemiological analysis of site relationships of synchronous and metachronous multiple primary cancers in the National Cancer Center, Japan, 1962-1996. Jpn J Clin Oncol 29: 96-105, 1999.

12. Sobin LH, Gospodarowicz MK, Wittekind C and International Union against Cancer: TNM classification of malignant tumours. Wiley-Blackwell, Chichester, West Sussex, UK; Hoboken, NJ, 2010.

13. National Cancer Institute (U.S.): Common terminology criteria for adverse events (CTCAE). U.S. Dept. of Health and Human Services, National Institutes of Health, National Cancer Institute, Bethesda, Md., 2009.

14. Warren S and Gates O: Multiple primary malignant tumors: A survey of the literature and statistical study. Am J Cancer 16: 1358-1414, 1932.

15. Németh Z, Czigner J, Iván L, Ujpál M, Barabás J and Szabó G: Quadruple cancer, including triple cancers in the head and neck region. Neoplasma 49: 412-414, 2002.

16. Moertel CG, Dockerty MB and Baggenstoss AH: Multiple primary malignant neoplasms. I. Introduction and presentation of data. Cancer 14: 221-230, 1961.

17. Vogt A, Schmid S, Heinimann K, Frick H, Herrmann C, Cerny T and Omlin A: Multiple primary tumours: Challenges and approaches, a review. ESMO Open 2: e000172, 2017.

18. Bartelink H, Roelofsen F, Eschwege F, Rougier P, Bosset JF, Gonzalez DG, Peiffert D, van Glabbeke M and Pierart M: Concomitant radiotherapy and chemotherapy is superior to radiotherapy alone in the treatment of locally advanced anal cancer: Results of a phase III randomized trial of the European Organization for Research and Treatment of Cancer Radiotherapy and Gastrointestinal Cooperative Groups. J Clin Oncol 15: 2040-2049, 1997.

19. Huang K, Haas-Kogan D, Weinberg V and Krieg R: Higher radiation dose with a shorter treatment duration improves outcome for locally advanced carcinoma of anal canal. World J Gastroenterol 13: 895-900, 2007.

20. Peiffert D, Tournier-Rangeard L, Gérard JP, Lemanski C, François E, Giovannini M, Cvitkovic F, Mirabel X, Bouché O, Luporsi $\mathrm{E}$, et al: Induction chemotherapy and dose intensification of the radiation boost in locally advanced anal canal carcinoma: Final analysis of the randomized UNICANCER ACCORD 03 trial. J Clin Oncol 30: 1941-1948, 2012.

21. Landoni F, Maneo A, Colombo A, Placa F, Milani R, Perego P, Favini G, Ferri L and Mangioni C: Randomised study of radical surgery versus radiotherapy for stage Ib-IIa cervical cancer. Lancet 350: 535-540, 1997.

22. Toita T, Kato S, Niibe Y, Ohno T, Kazumoto T, Kodaira T, Kataoka M, Shikama N, Kenjo M, Tokumaru S, et al: Prospective multi-institutional study of definitive radiotherapy with high-dose-rate intracavitary brachytherapy in patients with nonbulky $(<4-\mathrm{cm})$ stage I and II uterine cervical cancer (JAROG0401/JROSG04-2). Int J Radiat Oncol Biol Phys 82: e49-e56, 2012.

23. Brierley J, Gospodarowicz MK and Wittekind C (eds): TNM classification of malignant tumours. Wiley Blackwell, Chichester, West Sussex, UK; Hoboken, NJ, 2017.

24. Ajani JA, Winter KA, Gunderson LL, Pedersen J, Benson AB III, Thomas CR Jr, Mayer RJ, Haddock MG, Rich TA and Willett C: Fluorouracil, mitomycin, and radiotherapy vs fluorouracil, cisplatin, and radiotherapy for carcinoma of the anal canal: A randomized controlled trial. JAMA 299: 1914-1921, 2008.

25. Kachnic LA, Winter K, Myerson RJ, Goodyear MD, Willins J, Esthappan J, Haddock MG, Rotman M, Parikh PJ, Safran H and Willett CG: RTOG 0529: A phase 2 evaluation of dose-painted intensity modulated radiation therapy in combination with 5-fluorouracil and mitomycin- $\mathrm{C}$ for the reduction of acute morbidity in carcinoma of the anal canal. Int J Radiat Oncol Biol Phys 86: 27-33, 2013.

26. Jadon R, Pembroke CA, Hanna CL, Palaniappan N, Evans M, Cleves AE and Staffurth J: A systematic review of organ motion and image-guided strategies in external beam radiotherapy for cervical cancer. Clin Oncol (R Coll Radiol) 26: 185-196, 2014.

27. Taylor A and Powell ME: An assessment of interfractional uterine and cervical motion: Implications for radiotherapy target volume definition in gynaecological cancer. Radiother Oncol 88: 250-257, 2008

28. Zeng L, Ou G, Itasaka S, Harada H, Xie X, Shibuya K, Kizaka-Kondoh S, Morinibu A, Shinomiya K and Hiraoka M: TS-1 enhances the effect of radiotherapy by suppressing radiation-induced hypoxia-inducible factor-1 activation and inducing endothelial cell apoptosis. Cancer Sci 99: 2327-2335, 2008. 
29. Boku N, Yamamoto S, Fukuda H, Shirao K, Doi T, Sawaki A, Koizumi W, Saito H, Yamaguchi K, Takiuchi $\mathrm{H}$, et al: Fluorouracil versus combination of irinotecan plus cisplatin versus S-1 in metastatic gastric cancer: A randomised phase 3 study. Lancet Oncol 10: 1063-1069, 2009.

30. Takashima A, Shimada Y, Hamaguchi T, Ito Y, Nakano A, Nakamura K, Shibata T, Fukuda H and Moriya Y; Colorectal Cancer Study Group of the Japan Clinical Oncology Group: A Phase I/II trial of chemoradiotherapy concurrent with S-1 plus mitomycin $\mathrm{C}$ in patients with clinical Stage II/III squamous cell carcinoma of anal canal (JCOG0903: SMART-AC). Jpn J Clin Oncol 41: 713-717, 2011.

31. Li Z, Mao W, Lin N and Han S: Concurrent radiotherapy with S-1 plus cisplatin versus concurrent radiotherapy with cisplatin alone for the treatment of locally advanced cervical carcinoma: A pilot randomised controlled trial. Clin Transl Oncol 18: 413-417, 2016.
32. Schwarz JK, Lewis JS Jr, Pfeifer J, Huettner P and Grigsby P: Prognostic significance of p16 expression in advanced cervical cancer treated with definitive radiotherapy. Int J Radiat Oncol Biol Physics 84: 153-157, 2012.

33. Serup-Hansen E, Linnemann D, Skovrider-Ruminski W, Høgdall E, Geertsen PF and Havsteen H: Human papillomavirus genotyping and p16 expression as prognostic factors for patients with American Joint Committee on Cancer stages I to III carcinoma of the anal canal. J Clin Oncol 32: 1812-1817, 2014.

This work is licensed under a Creative Commons Attribution-NonCommercial-NoDerivatives 4.0 International (CC BY-NC-ND 4.0) License. 